\title{
Riesgo de resangrado en hemorragia digestiva alta según la escala de Glasgow-Blatchford: herramienta de triaje
}

Miroslava Olivarec-Bonilla, ${ }^{1}$ Anaidt M. García-Montano ${ }^{1}$ y Armando Herrera-Arellano ${ }^{2 *}$

${ }^{1}$ Instituto Mexicano del Seguro Social, Hospital General Regional 1, Servicio de Urgencias Adultos, Cuernavaca, Morelos; ${ }^{2}$ Universidad Autónoma del Estado de Morelos, Facultad de Medicina, Cuernavaca, Morelos. México

\section{Resumen}

Introducción: Con la escala de Glasgow-Blatchford (EG-B) se califica mediante datos clínicos, el riesgo de resangrado después de hemorragia del tubo digestivo alto (HTDA); y con las escalas de Forrest y Dagradi, mediante endoscopia. Objetivo: Evaluar la capacidad de la EG-B para identificar riesgo de resangrado a 30 días después de una HTDA; el estándar de oro de comparación fue la endoscopia. Método: Se analizaron 129 expedientes de pacientes con HTDA y endoscopia. Se cuantificaron las escalas de Glasgow-Blatchford, Forrest y Dagradi; se calculó sensibilidad, especificidad y área bajo la curva ROC (ABC-ROC) del riesgo de resangrado reportado por EG-B. Resultados: La EG-B identificó a 53 pacientes con riesgo bajo de resangrado (41.09\%) y 76 con riesgo alto (58.91\%). Con la endoscopia se identificó a 107 pacientes con hemorragia no variceal (82.94 \%), 98 con riesgo bajo (89.9\%) y 11 con riesgo alto (10.09\%); además, 22 pacientes con hemorragia variceal (17.05\%), 12 con riesgo bajo (54.54 \%) y 10 con riesgo alto (45.45\%). La EG-B mostró sensibilidad de 0.857, especificidad de 0.462 y ABC-ROC de 0.660. Conclusiones: $L a E G-B$ es sencilla, objetiva y útil para identificar riesgo de resangrado después de HTDA; se sugiere como herramienta de triaje en urgencias.

PALABRAS CLAVE: Hemorragia digestiva alta. Riesgo de resangrado. Endoscopia. Escala de Glasgow-Blatchford. Escala de Forrest. Escala de Dagradi.

\section{Upper gastrointestinal hemorrhage re-bleeding risk according to the Glasgow- Blatchford scale: a triage tool}

\section{Abstract}

Introduction: The Glasgow-Blatchford scale (GBS) classifies the risk of re-bleeding after upper gastrointestinal bleeding (UGIB) using clinical data, whereas the Forrest and Dagradi scales do it by endoscopy. Objective: To assess GBS's ability to identify re-bleeding risk within 30 days of an UGIB, using endoscopy as the gold standard for comparison. Method: 129 medical records of patients with UGIB and endoscopy were analyzed. The Glasgow-Blatchford, Forrest and Dagradi scales were quantified; sensitivity, specificity and area under the ROC curve (AUC-ROC) of GBS-reported re-bleeding risk were calculated. Results: GBS identified 53 patients with low re-bleeding risk (41.09\%) and 76 with high risk (58.91\%). Endoscopy identified 107 patients with non-variceal bleeding (82.94 \%): 98 with low risk (89.9\%) and 11 with high risk (10.09\%); in addition, it identified 22 patients with variceal hemorrhage (17.05\%): 12 with low risk (54.54\%) and 10 with high risk (45.45\%). GBS showed a sensitivity of 0.857 , specificity of 0.462 and an AUC-ROC of 0.660. Conclusions: GBS is simple, objective and useful to identify the risk of re-bleeding after UGIB; it is suggested as a triage tool in the emergency department.

KEY WORDS: Upper gastrointestinal hemorrhage. Re-bleeding risk. Endoscopy. Glasgow-Blatchford scale. Forrest scale. Dagradi scale.

Correspondencia:

*Armando Herrera-Arellano

E-mail: armandoha_mx@yahoo.com.mx

0016-3813/๑ 2020 Academia Nacional de Medicina de México, A.C. Publicado por Permanyer. Este es un artículo open access bajo la licencia CC BY-NC-ND (http://creativecommons.org/licenses/by-nc-nd/4.0/).

Fecha de recepción: 05-11-2019

Fecha de aceptación: 26-05-2020

DOI: $10.24875 / G M M .20005607$
A.C. Publicado por Permanyer. Este es un artículo open access bajo la licencia 


\section{Introducción}

La hemorragia del tubo digestivo alto (HTDA) es motivo frecuente de ingreso en los servicios de urgencias; se define como el sangrado gastrointestinal por arriba del ángulo de Treitz, de origen no variceal y variceal. Se ha observado hasta $16.5 \%$ de resangrado a 30 días del alta hospitalaria ${ }^{1}$ que requiere hasta 133368 días de hospitalización al año, con importante sobrecarga económica. ${ }^{2,3}$ En México, la mortalidad por HTDA oscila entre 3.3 y $6 \%$ y las varices esofágicas son la causa más común; ;-6 en otros países, la enfermedad ácido-péptica ocupa el primer lugar. ${ }^{7,8}$

El riesgo de la HTDA se estadifica con parámetros clínicos y endoscópicos que orientan la necesidad de hemotransfusión o cirugía, así como el riesgo de mortalidad, recidiva o persistencia del sangrado a 30 días. ${ }^{9}$ Las escalas pronósticas clasifican el riesgo de resangrado a 30 días en bajo y alto. La escala de Glasgow-Blatchford (EG-B) no requiere estudios endoscópicos y se integra con datos clínicos y de laboratorio; además, estratifica la necesidad de procedimientos diagnósticos y terapéuticos urgentes. ${ }^{1,10-12}$ Los pacientes calificados mediante EG-B con riesgo bajo de resangrado se pueden tratar en forma segura como ambulatorios y en los evaluados con riesgo alto se debe practicar endoscopia, preferentemente en las primeras 12 horas. $^{13}$

Ya que la endoscopia depende de la disponibilidad del recurso, el triaje con escalas permiten disminuir los ingresos hospitalarios en pacientes de riesgo bajo, así como la estancia prolongada. ${ }^{14}$ Para pronosticar resangrado y la necesidad de tratamiento endoscópico, transfusión o cirugía, la EG-B es igual de útil que la escala posendoscópica de Rockall y mejor que la preendoscópica de Rockall. ${ }^{15-19}$ En la EG-B, el punto de corte $\leq 2$ es el mejor predictor de riesgo bajo de resangrado y egreso seguro del paciente..$^{19-21}$

Las escalas endoscópicas identifican con mayor certeza el riesgo de resangrado a 30 días. En úlcera péptica, la escala de Forrest asigna las categorías IA, IB, IIA y IIB al riesgo alto, y las categorías IIC y III a riesgo bajo; ${ }^{22,23}$ mientras que en várices esofágicas, la de Dagradi identifica seis grados, de los cuales IV, $\mathrm{V}$ y $\mathrm{VI}$ indican riesgo alto. ${ }^{24-26}$ Aun cuando existen varias escalas validadas para identificar el riesgo de resangrado después de HTDA, solo se aplican en $19.4 \%$ de los casos. ${ }^{10,26,27}$
El objetivo de la investigación que se presenta fue identificar sensibilidad, especificidad y área bajo la curva ROC (ABC-ROC) de la EG-B para riesgo de resangrado a 30 días en pacientes con HTDA; el estándar de oro fue el riesgo determinado mediante endoscopia.

\section{Método}

Estudio transversal, observacional y analítico en el que se incluyeron expedientes de hombres y mujeres, con edad igual o mayor a 18 años, ingresados durante 2016 al Servicio de Urgencias del Hospital General Regional 1 del Instituto Mexicano del Seguro Social en Cuernavaca, Morelos, con HTDA de origen variceal y no variceal, confirmada por endoscopia en las 24 horas posteriores al ingreso. Se excluyeron expedientes con información incompleta para estadificar la EG-B, en los que existiera registro de diagnóstico de cáncer digestivo alto, enfermedades hemorrágicas, esófago de Barret, tratamiento anticoagulante y los correspondientes a casos que procedían de otro hospital. Las variables que identificaron el riesgo de resangrado a 30 días fueron las siguientes:

- EG-B: riesgo bajo $\leq 2$ puntos y riesgo alto $\geq 3$ puntos.

- Escala endoscópica de Forrest para valorar HTDA no variceal: riesgo bajo, clases IIC y III; riesgo alto, clases IA, IB, IIA y IIB.

- Escala endoscópica de Dagradi para HTDA variceal: riesgo bajo, clases I a III; riesgo alto, clases IV a VI. ${ }^{27,28}$

Se utilizó muestreo no probabilístico y la muestra se conformó con todos los expedientes de pacientes con HTDA durante 2016 que cumplieron con los criterios de selección. Para el análisis de datos se empleó estadística descriptiva; además, se calculó sensibilidad, especificidad, valores predictivos positivo y negativo, así como $A B C-R O C$ del riesgo de resangrado indicado por la EG-B, cuyo parámetro de referencia fue el riesgo endoscópico. El análisis se efectuó con el programa Stata versión 13. El comité local de investigación del hospital autorizó el protocolo con registro R-2017-1701-15.

\section{Resultados}

Durante 2016 se otorgaron 19111 consultas de urgencia en el Hospital General Regional 1 a pacientes mayores de 16 años e ingresaron 390 enfermos con HTDA, que representaron 20.4 casos de HTDA 
Tabla 1. Comparación de los grupos de riesgo de resangrado identificados mediante la escala de Glasgow-Blatchford*

\begin{tabular}{|c|c|c|c|c|c|}
\hline \multirow[t]{2}{*}{ Variable } & \multicolumn{2}{|c|}{ Riesgo bajo* ( $n=53$ ) } & \multicolumn{2}{|c|}{ Riesgo alto** $(n=76)$} & \multirow{2}{*}{$\frac{\text { t Student }}{p}$} \\
\hline & Media & DE & Media & DE & \\
\hline Edad & 62.94 & 16.42 & 63.78 & 15.55 & 0.76 \\
\hline Frecuencia cardiaca (latidos/minuto) & 77.79 & 7.86 & 88.51 & 15.80 & 0.0001 \\
\hline PAS $(\mathrm{mm} \mathrm{Hg})$ & 118.07 & 4.84 & 102.15 & 12.22 & 0.001 \\
\hline PAD (mm Hg) & 74.52 & 10.34 & 68.47 & 9.72 & 0.0009 \\
\hline Urea (mg/dL) & 22.22 & 12.79 & 55.79 & 28.29 & 0.0001 \\
\hline $\begin{array}{l}\text { Hemoglobina }(\mathrm{g} / \mathrm{dL}) \\
\text { Hombres }(\mathrm{g} / \mathrm{dL}) \\
\text { Mujeres }(\mathrm{g} / \mathrm{dL})\end{array}$ & $\begin{array}{l}13.12 \\
13.30 \\
13.00\end{array}$ & $\begin{array}{l}0.66 \\
0.69 \\
0.62\end{array}$ & $\begin{array}{l}11.63 \\
11.69 \\
11.54\end{array}$ & $\begin{array}{l}1.32 \\
1.14 \\
1.57\end{array}$ & $\begin{array}{l}0.0001 \\
0.0001 \\
0.0001\end{array}$ \\
\hline $\begin{array}{l}\text { EG-B (puntos) } \\
\text { HTDA no variceal } \\
\text { HTDA variceal }\end{array}$ & $\begin{array}{c}1.22 \\
1.22 \\
2\end{array}$ & $\begin{array}{l}0.92 \\
0.92 \\
-\end{array}$ & $\begin{array}{c}7.30 \\
6.63 \\
9.3\end{array}$ & $\begin{array}{l}3.26 \\
2.91 \\
4.21\end{array}$ & $\begin{array}{c}0.0001 \\
0.0001 \\
N / D\end{array}$ \\
\hline
\end{tabular}

por cada 1000 consultas al año. Con la revisión de los expedientes se identificaron 154 pacientes con HTDA y endoscopia en las primeras 24 horas (39.4\%); en 25 no se cumplieron los criterios de inclusión. Por lo anterior, en el análisis de datos se incluyeron 129 casos, que correspondieron a $33.1 \%$ del total de pacientes con HTDA ingresados en 2016.

En la muestra predominaron los hombres (52\%) y la edad promedio fue $63.4 \pm 15.86$ años; los principales antecedentes relacionados con HTDA fueron empleo crónico de antiinflamatorios no esteroideos (66.7\%), hepatopatía (38.7\%) y alcoholismo (32.03\%). Otras comorbilidades fueron artropatía (27.91\%), hipertensión arterial (27.13\%), enfermedad renal crónica $(12.4 \%)$ e insuficiencia cardiaca (3.88 \%). Los síntomas predominantes fueron hematemesis $(92.2 \%)$ y melena (46.5 \%).

Con la EG-B se obtuvo una media de $5.04 \pm 3.96$ puntos y se identificó a 53 pacientes con riesgo bajo de resangrado ( $41.09 \%$ ) y 76 con riesgo alto $(58.91 \%)$. En la Tabla 1 se detallan los promedios de las variables que integran la EG-B según el riesgo de resangrado, así como la puntuación obtenida en los casos con HTDA no variceal y variceal. Solo un paciente con HTDA variceal obtuvo riesgo bajo con la EGB. Con excepción de la presión arterial y la hemoglobina, en la mayoría de las variables se observaron diferencias significativas a favor del grupo con riesgo alto.

Mediante endoscopia se identificaron 107 casos con sangrado no variceal (82.94\%), 20 con sangrado variceal $(15.5 \%)$ y dos con sangrado mixto (1.55\%). Por lo anterior, el análisis incluyó 109 diagnósticos de sangrado no variceal y 22 de origen variceal. En los pacientes con HTDA no variceal se identificaron 84 con úlcera gástrica $(77.06 \%)$ y 25 con gastritis erosiva (22.94\%); con la escala de Forrest se identificaron 98 casos con riesgo bajo de resangrado (89.9\%) y 11 con riesgo alto (10.09\%). Además, con la escala de Dagradi se identificaron 12 casos con riesgo bajo de resangrado $(54.5 \%)$ y 10 con riesgo alto $(45.45 \%)$ en los casos de HTDA variceal (Tabla 2).

En la Tabla 3 se describe la sensibilidad y especificidad de la EG-B para identificar riesgo de resangrado a 30 días a partir del riesgo endoscópico como referencia; se indican los datos del total de la muestra y los de los grupos con HTDA no variceal y variceal. La sensibilidad mostró valores entre 0.818 y 0.900 ; el más alto correspondió a HTDA variceal. La especificidad en los tres grupos fue baja (entre 0 y 0.51 ). Conviene resaltar que el único caso de sangrado variceal clasificado con riesgo bajo mediante la EG-B resultó ser falso negativo, por lo que la especificidad en este grupo fue cero. En cuanto al ABC-ROC, los mejores resultados se observaron en el grupo de sangrado no variceal (0.664) y en la población total bajo estudio $(0.660)$ (Figuras 1 a 3). En la muestra no hubo defunciones durante la estancia en urgencias.

\section{Discusión}

La EG-B se ha empleado sistemáticamente en pacientes con HTDA como el mejor predictor de 
Tabla 2. Clasificación por endoscopia del riesgo de resangrado en pacientes con HTDA, según su origen: no variceal mediante escala de Forrest y variceal mediante escala de Dagradi

\begin{tabular}{|c|c|c|c|c|}
\hline Escala de Forrest & Riesgo & Frecuencia & $(\%)$ & Acumulado (\%) \\
\hline $\begin{array}{l}\text { Sangrado activo } \\
\text { IA } \\
\text { IB }\end{array}$ & $\begin{array}{l}\text { Alto } \\
\text { Alto }\end{array}$ & $\begin{array}{l}2 \\
6\end{array}$ & $\begin{array}{l}1.83 \\
5.50\end{array}$ & $\begin{array}{l}1.83 \\
7.33\end{array}$ \\
\hline $\begin{array}{l}\text { Sangrado reciente } \\
\text { IIA } \\
\text { IIB } \\
\text { IIC }\end{array}$ & $\begin{array}{l}\text { Alto } \\
\text { Alto } \\
\text { Bajo }\end{array}$ & $\begin{array}{c}2 \\
1 \\
11\end{array}$ & $\begin{array}{c}1.83 \\
0.92 \\
10.10\end{array}$ & $\begin{array}{c}9.16 \\
10.08 \\
20.18\end{array}$ \\
\hline $\begin{array}{l}\text { Sin sangrado } \\
\text { III } \\
\text { Total }\end{array}$ & Bajo & $\begin{array}{c}87 \\
109\end{array}$ & $\begin{array}{l}79.81 \\
99.99\end{array}$ & 99.99 \\
\hline Escala de Dagradi & Riesgo & Frecuencia & $(\%)$ & Acumulado (\%) \\
\hline I & Bajo & 3 & 13.64 & 13.64 \\
\hline$\|$ & Bajo & 1 & 4.54 & 18.18 \\
\hline III & Bajo & 8 & 40.91 & 59.09 \\
\hline IV & Alto & 0 & 0.00 & 59.09 \\
\hline v & Alto & 6 & 22.72 & 81.81 \\
\hline $\mathrm{VI}$ & Alto & 4 & 18.18 & 99.99 \\
\hline Total & & 22 & 99.99 & \\
\hline
\end{tabular}

Tabla 3. Valores de la prueba diagnóstica de la EG-B para predecir riesgo de resangrado en pacientes con HTDA*

\begin{tabular}{|l|c|c|c|c|c|}
\hline Grupo & Sensibilidad & Especificidad & VP+ & VP- & ABC-ROC \\
\hline HTDA toda la muestra & 0.857 & 0.462 & 0.236 & 0.943 & 0.660 \\
\hline HTDA no variceal & 0.818 & 0.510 & 0.157 & 0.961 & 0.664 \\
\hline HTDA variceal & 0.900 & 0.00 & 0.428 & 0.00 & 0.500
\end{tabular}

*El estándar de oro fue el riesgo reportado mediante endoscopia. EG-B = escala de Glasgow-Blatchford, HTDA = hemorragia del tubo digestivo alto, VP+ = valor predictivo positivo, $\mathrm{VP}-=$ valor predictivo negativo, $\mathrm{ABC}-\mathrm{ROC}$ = área bajo la curva $\mathrm{ROC}$.

resangrado a 30 días, de necesidad de endoscopia urgente, intervención endoscópica y muerte. ${ }^{15,17-19}$ Por lo anterior, en el consenso para el manejo de pacientes con HTDA no variceal se recomienda estratificar el riesgo de resangrado y de mortalidad con escalas ya validadas, practicar endoscopia durante las primeras 24 horas en la mayoría de los pacientes y egresar los casos clasificados mediante examen clínico o endoscópico con riesgo bajo de resangrado. ${ }^{9}$

Conforme nuestro conocimiento, solo existen dos publicaciones acerca de pacientes con HTDA ingresados en urgencias de hospitales mexicanos; solo en uno se emplearon escalas pronósticas. ${ }^{4,25}$ Lo anterior indica que en la mayoría de los hospitales en México no se aplican rutinariamente escalas validadas para asignar el riesgo de resangrado o muerte en los pacientes con HTDA, aun cuando es incierta la disponibilidad de la endoscopia. Lo anterior dificulta determinar el diagnóstico de certeza, los riesgos reales y el tratamiento adecuado de la HTDA. Esta falta de identificación objetiva de riesgos origina ingresos innecesarios de pacientes con riesgo bajo y, en el peor de los casos, egresos de pacientes con riesgo alto de desenlace grave o fatal.

La HTDA se clasifica, según su origen, en sangrado variceal (asociado principalmente a hipertensión portal) y no variceal (asociado principalmente a enfermedad ácido-péptica). En nuestro estudio predominaron los hombres (52\%), dato similar a $51 \%$ reportado en otra investigación en México, pero diferente a $44 \%$ identificado en China. ${ }^{4,8}$ El promedio de edad de nuestros 


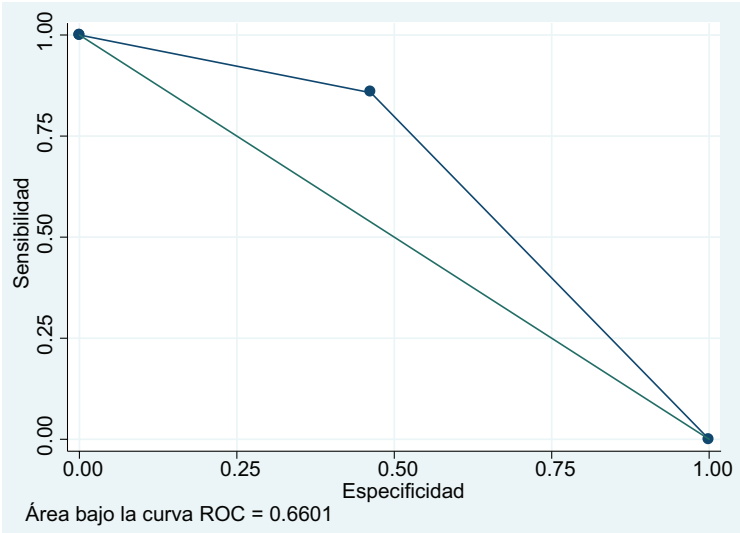

Figura 1. Sensibilidad, especificidad y área bajo la curva ROC de la escala Glasgow-Blatchford para identificar riesgo de resangrado a 30 días en la muestra total de pacientes con HTDA; el estándar de oro fue el riesgo reportado por endoscopia.

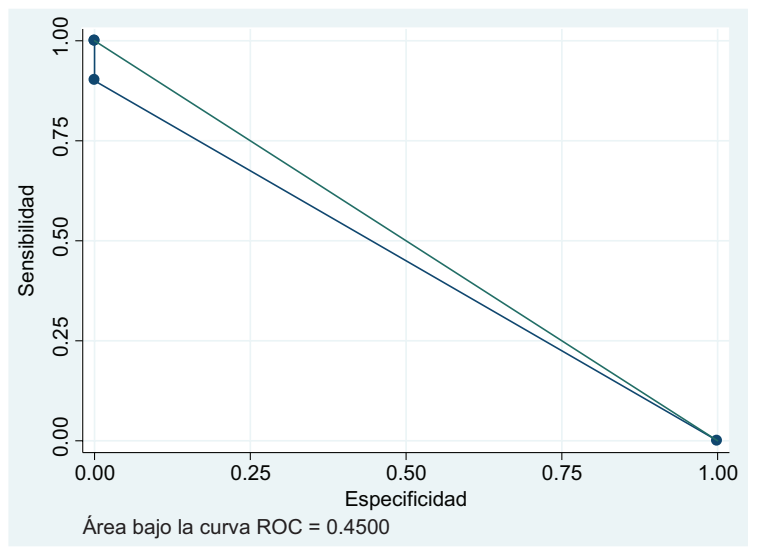

Figura 3. Sensibilidad, especificidad y área bajo la curva ROC de la escala Glasgow-Blatchford para identificar riesgo de resangrado a 30 días en los casos de HTDA variceal; el estándar de oro fue el riesgo reportado por endoscopia.

pacientes fue $63.4 \pm 15.86$ años, que concuerda con la media de 61.3 años reportada por Budimir en 2016 en pacientes con sangrado variceal, pero mayor a 51.5 años indicada por Wang en 2017 y por Moctezuma Maldonado en $2008.4,8,28$ Por otro lado, ya que predominó la HTDA no variceal era de esperar que la mayoría de casos indicara uso crónico de antiinflamatorios no esteroideos $(67 \%$ ) y que predominaran la hematemesis (92\%) y la melena (46\%). Estos datos son discordantes con los de dos estudios en México que reportaron porcentajes más bajos de uso de antiinflamatorios no esteroideos, hematemesis y melena. ${ }^{4,5}$

Para evaluar el riesgo de resangrado a 30 días consideramos un valor de 2 como punto de corte en

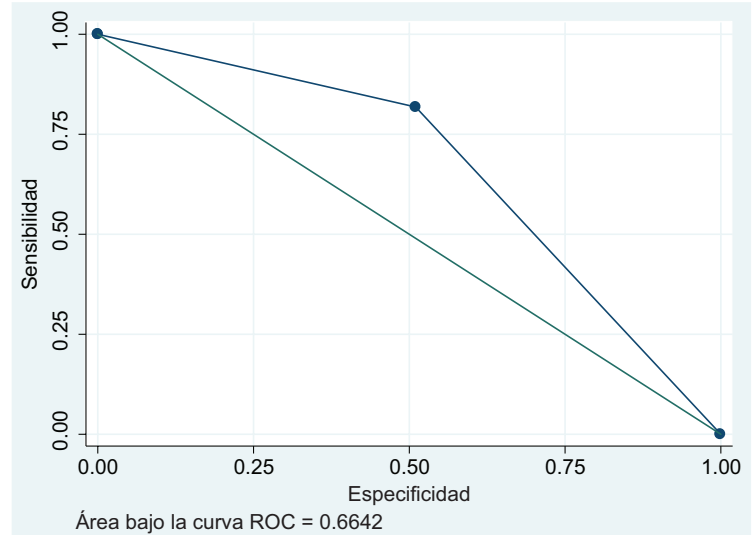

Figura 2. Sensibilidad, especificidad y área bajo la curva ROC de la escala Glasgow-Blatchford para identificar riesgo de resangrado a 30 días en los casos de HTDA no variceal; el estándar de oro fue el riesgo reportado por endoscopia.

la EG-B. Con base en esto, en la muestra identificamos $41 \%$ de casos con riesgo bajo y $59 \%$ con riesgo alto (sensibilidad de 0.857 , especificidad de $0.462 \mathrm{y}$ ABC-ROC de 0.660). Este mismo punto de corte lo adoptaron Le Jeune (2011) y Recio-Ramírez (2015), quienes mostraron que una puntuación $\leq 2$ indica baja probabilidad de resultados clínicos adversos a 30 días, incluido el riesgo de resangrado, y lo recomiendan para el egreso temprano. ${ }^{19,24}$ Sin embargo, otros autores emplearon una puntuación $>1$ para identificar el riesgo alto. Así, Mustafa en 2015 reportó $64.4 \%$ con riesgo alto y $35.6 \%$ con riesgo bajo; de estos últimos, casi la mitad egresó sin ningún desenlace adverso. ${ }^{18}$ En 2016, Motola Kuba indicó que la EG-B es el mejor predictor de resangrado en pacientes con HTDA variceal por cirrosis hepática y que indicó mayor $\mathrm{ABC}-\mathrm{ROC}$ que la escala preendoscópica de Rockall; concluyó que el empleo rutinario de la escala evita ingresos o procedimiento innecesarios e incrementa los egresos prematuros y seguros. ${ }^{21}$

En 2016, en el hospital sede, la endoscopia se realizó durante las primeras 24 horas a $40 \%$ de todos los casos de HTDA admitidos en el Servicio de Urgencias, independientemente de la evaluación objetiva del riesgo de resangrado. Diversos autores mencionan que la endoscopia realizada después de 24 horas se asocia con aumento de la mortalidad, la estancia hospitalarias y los costos. ${ }^{17}$ En la muestra analizada, a $17 \%$ de los casos se les practicó endoscopia durante las primeras 12 horas; en contraposición, en otra investigación en un hospital particular de la Ciudad de México donde se dispone de 
endoscopia en forma continua, se informó que se realizó este estudio a $98 \%$ de los casos de HTDA. ${ }^{4}$ En nuestro análisis, por endoscopia se identificó a $83.7 \%$ de los casos de sangrado no variceal, dato similar al de otro estudio en México en el que se registró enfermedad ácido péptica (úlceras y erosiones) en $90 \%$ de los casos. ${ }^{4}$ Sin embargo, nuestro porcentaje de casos de HTDA no variceal difiere con lo reportado en poblaciones de China y África, donde 52.7 y $51.3 \%$ presentó este tipo de HTDA. ${ }^{11,25}$ Sin embargo, en relación con la HTDA variceal, el estudio en China indica porcentajes similares a los nuestros (15.1 y $17 \%)$. $^{11}$

En la mayoría de los casos se determinó riesgo bajo de resangrado mediante escalas endoscópicas: $89.9 \%$ en HTDA no variceal y $54.54 \%$ en HTDA variceal; en estos últimos, el hallazgo más frecuente fue varices esofágicas grado III (de 3 a $4 \mathrm{~mm}$ de diámetro, prominentes y tortuosas), seguidas de los grados IV y V (> $5 \mathrm{~mm}$ y con signos rojos, variables relacionadas con riesgo alto de resangrado).

En nuestro estudio, la EG-B mostró alta sensibilidad (0.857) y buena especificidad (0.462) para predecir alto riesgo de resangrado a 30 días, independientemente del origen del sangrado $(\mathrm{ABC}-\mathrm{ROC}=0.660)$; también mostró alta sensibilidad para identificar riesgo alto de resangrado en pacientes con HTDA variceal $(0.900)$ y no variceal $(0.818)$. Los mejores valores predictivos se obtuvieron en la muestra total y en HTDA no variceal (ABC-ROC de 63.5 y $62.5 \%$, respectivamente). En HTDA variceal, la EG-B mostró nula sensibilidad porque el único caso con riesgo bajo resultó ser falso negativo.

El valor predictivo negativo de la EG-B fue alto en la muestra total (0.943) y en HTDA no variceal (0.961), de tal forma se espera que más de $90 \%$ de estos pacientes con HTDA y EG-B de riesgo bajo no resangren en el siguiente mes. En estos mismos grupos, los valores predictivos positivos fueron bajos: 0.236 en la muestra total y 0.157 en HTDA no variceal, lo cual indica que ante una puntuación $\geq 3$ en la EG-B en pacientes con HTDA existe $23 \%$ de riesgo de resangrado a 30 días. En estos casos, para confirmar el origen de la HTDA y el riesgo de resangrado sugerimos practicar endoscopia durante las primeras 24 horas.

Concluimos que la EG-B es una herramienta sencilla, objetiva y útil para identificar el riesgo de resangrado a 30 días en pacientes con HTDA, independientemente del origen. Se recomienda como herramienta valiosa del triaje en los servicios de urgencias.

\section{Conflicto de intereses}

Los autores declaran no tener conflictos de intereses.

\section{Financiamiento}

Los autores no recibieron patrocinio para elaborar este artículo.

\section{Responsabilidades éticas}

Protección de personas y animales. Los autores declaran que para esta investigación no se realizaron experimentos en seres humanos ni en animales.

Confidencialidad de los datos. Los autores declaran que en este artículo no aparecen datos de pacientes.

Derecho a la privacidad y consentimiento informado. Los autores declaran que en este artículo no aparecen datos de pacientes.

\section{Bibliografía}

1. Strömdahl M, Helgeson J, Kalaitzakis E. Emergency readmission following acute upper gastrointestinal bleeding. Eur J Gastroenterol Hepatol. 2016;51:1375-1379.

2. Abougergi MS, Peluso H, Saltzman JR. Thirty-day readmission among patients with non-variceal upper gastrointestinal hemorrhage and effects on outcomes. Gastroenterology. 2018;155:38-46.

3. Adam V, Barkun A. Estimates of costs of hospital stay for variceal and nonvariceal upper gastrointestinal bleeding in the United States. Value Health. 2008;11:1-3.

4. Moctezuma-Maldonado U, Almeda-Valdez P, Lizardi-Cervera J, Morán-Consuelos MA, García-Corona $\mathrm{Cl}$. Hemorragia de tubo digestivo alto. Experiencia en el hospital Médica Sur. Rev Invest Med Sur Mex. 2008;15:176-184.

5. Cruz-Guillén AA, Sánchez-Espinosa JR. Presentación sindromatica del sangrado digestivo alto variceal y no-variceal en el servicio de urgencias de un hospital público de segundo nivel del estado de Chiapas. Análisis descriptivo y comparativo con la prevalencia nacional. Med Int Mex. 2013:29:449-457.

6. Miilunpohja S, Jyrkkä J, Kärkkäinen JM, Kastarinen H, Heikkinen M, Paajanen $\mathrm{H}$, et al. Long-term mortality and causes of death in endoscopically verified upper gastrointestinal bleeding: comparison of bleeding patients and population controls. Scand J Gastroenterol. 2017;52:1211-1218.

7. Hreinsson JP, Kalaitzakis E, Gudmundsson S, Björnsson ES. Upper gastrointestinal bleeding: incidence, etiology and outcomes in a population-based setting. Scand J Gastroenterol. 2013;48:439-447.

8. Wang J, Cui Y, Wang J, Chen B, He Y, Chen M. Clinical epidemiological characteristics and change trend of upper gastrointestinal bleeding over the past 15 years. Zhonghua Wei Chang Wai Ke Za Zhi. 2017;20:425-431.

9. Barkun AN, Bardou M, Kuipers EJ, Sung J, Hunt RH, Martel M, et al. International consensus recommendations on the management of patients with nonvariceal upper gastrointestinal bleeding. Ann Intern Med. 2010;152:101-113

10. Faroog FT, Lee MH, Das A, Dixit R, Wong RCK. Clinical triage decision vs risk scores in predicting the need for endotherapy in upper gastrointestinal bleeding. Am J Emerg Med. 2012;30:129-134.

11. Blanco-Vela Cl, Peláez-Luna M, Hernández-Guerrero Al, Martínez-García CL, Blanco-Velasco G, Zamora-Nava LE, et al. Guías de diagnóstico y manejo endoscópico de la hemorragia de tubo digestivo alto no-variceal. Asociación Mexicana de Endoscopia Gastrointestinal. Endoscopia. 2017:29:173-195.

12. Cai JX, Saltzman JR. Initial assessment, risk stratification, and early management of acute nonvariceal upper gastrointestinal hemorrhage. Gastrointest Endosc Clin N Am. 2018;28:261-275. 
13. Jeong N, Kim KS, Jung YS, Kim T, Shin SM. Delayed endoscopy is associated with increased mortality in upper gastrointestinal hemorrhage. Am J Emerg Med. 2019;37:277-280

14. Bryant RV, Kuo P, Williamson K, Yam C, Schoeman MN, Holloway RH et al. Performance of the Glasgow-Blatchford score in predicting clinical outcomes and intervention in hospitalized patients with upper GI bleeding. Gastrointest Endosc. 2013;78:576-583.

15. Le Jeune IR, Gordon AL, Farrugia D, Manwani R, Guha IN, James MW. Safe discharge of patients with low-risk upper gastrointestinal bleeding (UGIB): can the use of Glasgow-Blatchford Bleeding Score be extended? Acute Med. 2011;10:176-181.

16. Waddell KM, Stanley AJ. Risk assessment scores for patients with upper gastrointestinal bleeding and their use in clinical practice. Hosp Pract (1995). 2015;43:290-298.

17. Tang Y, Shen J, Zhang F, Zhou X, Tang Z, You T. Scoring systems used to predict mortality in patients with acute upper gastrointestinal bleeding in the ED. Am J Emerg Med. 2018;36:27-32.

18. Mustafa Z, Cameron A, Clark E, Stanley AJ. Outpatient management of low-risk patients with upper gastrointestinal bleeding: can we safely extend the Glasgow Blatchford Score in clinical practice? Eur J Gastroenterol Hepatol. 2015;27:512-515.

19. Recio-Ramírez JM, Sáchez-Sáchez MP, Peña-Ojeda JA, Fernández-Romero E, Aguilera-Peña M, del-Campo-Molina E, et al. The predictive capacity of the Glasgow-Blatchford score for the risk stratification of upper gastrointestinal bleeding in an emergency department. Rev Esp Enferm Dig. 2015;107:262-267.

20. Sengupta N, Tapper EB, Patwardhan VR, Ketwaroo GA, Thaker AM Leffler DA, et al. High Glasgow Blatchford Score at admission is associated with recurrent bleeding after discharge for patients hospitalized with upper gastrointestinal bleeding. Endoscopy. 2016;48:9-15.
21. Stanley AJ, Laine L, Dalton HR, Ngu JH, Schultz M, Abazi R, et al. Comparison of risk scoring systems for patients presenting with upper gastrointestinal bleeding: international multicentre prospective study. BMJ. 2017;356:i6432.

22. De Groot NL, van Oijen MG, Kessels K, Hemmink M, Weusten BL, Timmer R, et al. Reassessment of the predictive value of the Forrest classification for peptic ulcer rebleeding and mortality: can classification be simplified? Endoscopy. 2014;46:46-52.

23. Noda H, Ogasawara N, Izawa S, Ozeki T, Yanamoto K, Okaniwa N, et al. Risk factors for bleeding evaluated using the Forrest classification in Japanese patients after endoscopic submucosal dissection for early gastric neoplasm. Eur J Gastroenterol Hepatol. 2015;27:1022-1029.

24. Narváez-Rivera RM, Cortez-Hernández CA, González-González JA, Tamayo-de la Cuesta JL, Zamarripa-Dorsey $F$, Torre-Delgadillo A, et al. Consenso Mexicano de Hipertensión Portal. Rev Gastroenterol Mex. 2013:78:92-113.

25. Motola-Kuba M, Escobedo-Arzate A, Tellez-Avila F, Altamirano J, Aguilar-Olivos N, González-Angulo A, et al. Validation of prognostic scores for clinical outcomes in cirrhotic patients with acute variceal bleeding. Ann Hepatol. 2016;15:895-901.

26. Santiago-Martínez E, Rodríguez-Magallán A. Indicadores clínicos, endoscópicos y de ultrasonido para riesgo de hemorragia variceal en pacientes con cirrosis. Rev Hosp Juarez Mex. 2006;73:44-50.

27. Leiman DA, Mills AM, Shofer FS, Weber AT, Leiman ER, Riff BP, et al. Glasgow Blatchford Score of limited benefit for low-risk urban patients: a mixed methods study. Endosc Int Open. 2017:5:E950-E958.

28. Budimir I, Gradišer M, Nikolić M, Baršić N, Ljubičić N, Kralj D, et al. Glasgow Blatchford, pre-endoscopic Rockall and AIMS65 scores show no difference in predicting rebleeding rate and mortality in variceal bleeding. Scand J Gastroenterol. 2016;51:1375-1379. 